This is an electronic reprint of the original article. This reprint may differ from the original in pagination and typographic detail.

Author(s): Airaksinen, Tuomas; Aittokoski, Timo

Title: $\quad$ Multi-objective actuator placement optimization for local sound control evaluated in a stochastic domain

Year: $\quad 2013$

Version:

Please cite the original version:

Airaksinen, T. \& Aittokoski, T. (2013). Multi-Objective Actuator Placement Optimization for Local Sound Control Evaluated in a Stochastic Domain. In: Repin, S., Tiihonen, T., Tuovinen, T. (eds.), Numerical Methods for Differential Equations, Optimization, and Technological Problems. Dedicated to Professor P. Neittaanmäki on His 60th Birthday. Computational Methods in Applied Sciences, 2013, Volume 27, Part 4, 321-334, DOI 10.1007/978-94-007-5288-7_17

All material supplied via JYX is protected by copyright and other intellectual property rights, and duplication or sale of all or part of any of the repository collections is not permitted, except that material may be duplicated by you for your research use or educational purposes in electronic or print form. You must obtain permission for any other use. Electronic or print copies may not be offered, whether for sale or otherwise to anyone who is not an authorised user. 


\title{
Multi-Objective Actuator Placement Optimization for Local Sound Control Evaluated in a Stochastic Domain
}

\author{
Tuomas Airaksinen and Timo Aittokoski
}

\begin{abstract}
A method to find optimal locations and properties of anti-noise actuators in a local noise control system is considered. The local noise control performance is approximated by an approach based on a finite element method, attempting to estimate the average performance of an optimal active noise control (ANC) system. Local noise control uses a fixed number of circular actuators that are located on the boundary of a three-dimensional enclosed acoustic space. Actuator signals are used to minimize the known harmonic noise at specified locations. The average noise reduction is maximized at two frequency ranges by adjusting the anti-noise actuator configuration, which is a non-linear multi-objective optimization problem. To solve the optimization problem, an unsorted population size evolutionary optimization algorithm (UPS-EMOA) is considered, and its performance is compared to the widely-known NSGA-II method. As a numerical example problem, the ANC in a passenger car cabin is considered. Significantly better noise control is obtained with the optimized actuator locations than only by a engineer's sophisticated guess.
\end{abstract}

\section{Introduction}

Noise generated by different machines is an increasing problem in modern working environments. Wheels, engines, and cooler fans are typical noise sources. There is an obvious need for noise control applications in factory environments, engineering vehicles, and passenger cars, for example. Sometimes it is possible to remove or reduce important noise source mechanisms by suitable design choices, which makes particular noise control approaches unnecessary. In many cases, however, this is not possible or the design is limited by other more important factors than noise.

Department of Mathematical Information Technology, University of Jyväskylä, P.O. Box 35 (Agora), FI-40014 University of Jyväskylä, Finland, e-mail: tuomas.airaksinen@jyu.fi, timo.aittokoski@jyu.fi 
Passive noise control techniques such as absorbing and insulating acoustic elements are effective methods in reducing high frequency sound, whereas active noise control (ANC) methods [11] are good at reducing low-frequency noise. The ANC is based on generating anti-sound with actuators. So the original noise is attenuated. In order to cancel the noise perfectly, the anti-sound must have the same amplitude as the noise, but an opposite phase so that destructive interference occurs. Local noise control employs ANC methods so that noise is reduced locally in a desired subdomain.

The most important frequencies originating in the passenger car engine are below $500 \mathrm{~Hz}$ [14]. As there are significant low-frequency noise sources, the local sound control can provide a significant noise reduction to the car cabin environment. Advanced methods designing and assessing such systems employ numerical simulation and optimization. Approaches using finite element modeling are presented in the articles $[15,5,13,1]$ of which $[5,13]$ consider also optimizing locations for anti-noise actuators.

In [1], a numerical evaluation method is developed for optimal local noise control, based on finite element modeling. The method determines the optimal performance of a local sound control by including the stochasticity of the cavity domain in the model. The anti-noise is optimized by minimizing the expected value of the noise computed using the finite element method. In this paper, this method is used to develop a technique to find optimal locations for anti-noise actuators. The optimization of actuator configuration is formulated as a multi-objective optimization problem such that optimal noise reduction at appropriate frequency ranges forms objective functions. By solving a multi-objective optimization problem, a whole family of Pareto-optimal solutions is obtained. An unrestricted population-size evolutionary multi-objective algorithm (UPS-EMOA, [3]) is used to solve the multi-objective optimization problem, and its performance is also compared to a well-known elitist non-dominated sorting genetic algorithm (NSGA-II, [8]).

This article is organized as follows. In Section 2, a mathematical model of sound propagation, the Helmholtz partial differential equation, and a numerical method to solve it are briefly presented. In Section 3, the local noise control in a stochastic domain is formulated as a quadratic optimization problem and an example of local noise control in a car driver's ears is described. The objective functions are also derived to evaluate actual anti-noise configurations. In Section 4 , the multiobjective optimization methods used in actuator configuration optimization are described briefly and the used parameters are given. In Section 5, the numerical results of actuator configuration optimization in a three-dimensional car cabin problem are studied and analyzed. Finally, in Section 6, conclusions are given.

\section{An Acoustic Model}

The time harmonic sound propagation is modeled by the Helmholtz equation 


$$
-\nabla \cdot \frac{1}{\rho} \nabla p-\frac{\omega^{2}}{c^{2} \rho} p=0 \quad \text { in } \Omega,
$$

where $\rho(\mathbf{x})$ is the density of the material at the location $\mathbf{x}$, and $c(\mathbf{x})$ is the speed of sound in the material. The complex pressure $p(\mathbf{x})$ defines the amplitude and phase of the pressure. The sound pressure at time $t$ is obtained by $\mathfrak{R e}\left(e^{-i \omega t} p\right)$, where $\omega$ is the angular frequency of sound and $i=\sqrt{-1}$. A sound source $f$ acting on a part $S$ of the boundary $\partial \Omega$ is modeled via a boundary condition. A partially absorbing wall material is described by the impedance boundary conditions

$$
\begin{array}{ll}
\frac{\partial p}{\partial \mathbf{n}}=\frac{i \eta \omega}{c} p+f & \text { on } S \\
\frac{\partial p}{\partial \mathbf{n}}=\frac{i \eta \omega}{c} p & \text { on } \partial \Omega \backslash S,
\end{array}
$$

where $\eta(\mathbf{x})$ is the absorption coefficient depending on the properties of the surface material. The value $\eta=1$ approximates a perfectly absorbing material and the value $\eta=0$ approximates a sound-hard material (the Neumann boundary condition).

An approximate solution for the partial differential equation (PDE) (1) can be obtained using a finite element method [16]. The finite element discretization transforms (1) into a system of linear equations $\mathbf{A x}=\mathbf{b}$, where the matrix $\mathbf{A}$ is generally symmetric, large, and sparse. Due to the large size and structure of $\mathbf{A}$, direct solution methods are computationally too expensive. Instead, an iterative solution method like GMRES needs to be used. Solving the system with a reasonable number of iterations is, however, challenging as the matrix $\mathbf{A}$ is badly conditioned and especially so when the calculation domain is large and the frequency is high. In the numerical example in Section 5, the solutions are computed after the systems are preconditioned by a damped Helmholtz preconditioner [2].

\section{The Noise Control Problem}

\subsection{Anti-Noise Actuator Signal Optimization}

The noise control problem is next presented briefly. A more detailed description is given in an earlier paper [1]. The problem is considered in the frequency domain, i.e. noise control is considered for one frequency at once; it should, however, be noted that the noise is not restricted to a single-frequency sound. The acoustic model is considered in an enclosed stochastic domain $\Omega(\mathbf{r})$, where $\mathbf{r}$ is a random vector that conforms to a known probability distribution $F(\mathbf{r})$. The sound pressure $p(\omega, \mathbf{x}, \mathbf{r}, \gamma)$ at an angular frequency $\omega$ is the sum of the sound pressures caused by noise and $n$ anti-noise sources 


$$
p(\omega, \mathbf{x}, \mathbf{r}, \gamma)=p_{0}(\omega, \mathbf{x}, \mathbf{r})+\sum_{j=1}^{n} \gamma_{j} p_{j}(\omega, \mathbf{x}, \mathbf{r})
$$

where the pressure amplitude $p_{0}$ is due to the noise source, $p_{j}$ is due to the $j$ th antinoise source, and $\gamma_{j}$ is a complex coefficient defining the amplitude and phase of the $j$ th anti-noise source. The noise and anti-noise sources are located on the boundaries of $\Omega$. The anti-noise defined by the coefficients $\gamma_{j}$ is optimized so that the noise is minimized in a subdomain denoted by $\Xi(\mathbf{r}) \subset \Omega(\mathbf{r})$. For this, a noise measure is defined as

$$
N(\omega, \mathbf{r}, \gamma)=\int_{\Xi(\mathbf{r})}|p(\omega, \mathbf{x}, \mathbf{r}, \gamma)|^{2} g(\mathbf{x}) d \mathbf{x}=\int_{\Xi(\mathbf{r})} p(\omega, \mathbf{x}, \mathbf{r}, \gamma) p(\omega, \mathbf{x}, \mathbf{r}, \gamma) g(\mathbf{x}) d \mathbf{x}
$$

where $g(\mathbf{x})$ is a weighting function and $p$ is the complex conjugate of $p$. The expected value of the noise measure in the stochastic domain $\Omega$ is given by

$$
E(N(\omega, \mathbf{r}, \gamma))=\int N(\omega, \mathbf{r}, \gamma) F(\mathbf{r}) d \mathbf{r}
$$

where $F(\mathbf{r})$ is the probability distribution of $\mathbf{r}$.

The objective function $J$ for optimization of the noise control problem for the single frequency $\omega$ is chosen to be an approximation of the integral (5) and it is given by the numerical quadrature

$$
J(\omega, \gamma)=\sum_{j=1}^{m} w_{j} N\left(\omega, \mathbf{r}_{j}, \gamma\right) F\left(\mathbf{r}_{j}\right)
$$

where the pairs $\left(\mathbf{r}_{j}, w_{j}\right)$ give the quadrature points and weights. The optimization problem is defined as

$$
\min _{\gamma \in \Gamma} J(\omega, \gamma)
$$

where $\Gamma$ is the set of feasible controls, which, for simplicity, is here $\Gamma=\mathbb{C}^{n}$. The optimal complex coefficients $\gamma_{i}$ that give phases and amplitudes for anti-noise actuators are now given by the optimality condition $\nabla_{\gamma} J=\mathbf{0}$, which leads to a system of linear equations.

\subsection{Anti-Noise Actuator Configuration Quality Measure}

The actual configuration of anti-noise actuators, i.e. their number, locations and other properties such as size, determine the performance that can be obtained for a local noise control system.

Let us first define another noise measure function 


$$
\tilde{N}(\omega, \mathbf{a}, \mathbf{r}, \gamma)=\int_{\Xi(\mathbf{r})}|p(\omega, \mathbf{a}, \mathbf{x}, \mathbf{r}, \gamma)| g(\mathbf{x}) d \mathbf{x}
$$

where $\mathbf{a}=\left(x_{1}, y_{1}, r_{1}, \cdots, x_{n}, y_{n}, r_{n}\right)$ is the anti-noise actuator configuration vector with $\left(x_{i}, y_{i}\right)$ determining the location and $r_{i}$ the radius of the $i$ th anti-noise actuator. Noise reduction for the frequency $\omega$ in $\mathrm{dB}$ is now

$$
R(\omega)=10 \log _{10} \frac{E\left(\tilde{N}\left(\omega, \mathbf{r}, \gamma_{\text {opt }}\right)\right)}{E(\tilde{N}(\omega, \mathbf{r}, \mathbf{0}))}=10 \log _{10} \frac{\int \tilde{N}\left(\omega, \mathbf{r}, \gamma_{\text {opt }}\right) F(\mathbf{r}) d \mathbf{r}}{\int \tilde{N}(\omega, \mathbf{r}, \mathbf{0}) F(\mathbf{r}) d \mathbf{r}},
$$

for $\gamma_{o p t}$ which is optimized according to (7). The quality measure of anti-noise actuator configuration at the frequency $\omega$ is obtained by replacing integrals in (9) with trapezoidal quadratures

$$
Q(\mathbf{a}, \omega)=10 \log _{10} \frac{\sum_{j} w_{j}^{\mathbf{r}} \tilde{N}\left(\omega, \mathbf{a}, \mathbf{r}_{j}, \gamma_{o p t}\right) F\left(\mathbf{r}_{j}\right)}{\sum_{j} w_{j}^{\mathbf{r}} \tilde{N}\left(\omega, \mathbf{r}_{j}, \mathbf{a}, \mathbf{0}\right) F\left(\mathbf{r}_{j}\right)}
$$

where $w_{j}^{\mathbf{r}}$ is quadrature weight from the trapezoidal rule for the integral of the probability distribution function $F$, and $\mathbf{r}_{j}$ is the co-ordinate triplet of the $j$ th quadrature point.

\subsection{Numerical Integration over Actuator}

Circle-shaped anti-noise actuators are placed on a subdomain of a boundary surface, which is denoted by $A \subset \partial \Omega$. The subdomain $A$ is composed of subdomains $A_{i}$ such that $A=\cup_{i} A_{i}$. In order to allow convenient implementation of anti-noise actuator configuration optimization, a geometrical linear mapping is defined from the two-dimensional rectangular plane-domain $\tilde{A}=\cup_{i} \tilde{A}_{i}$ to $A$, such that the subdomains $\tilde{A}_{i}$ are mapped to $A_{i}$, respectively. Integrals are approximated by using a finite element solution on a triangular mesh. In order to improve integration accuracy of the boundary line, the triangles that reside on the anti-noise actuator boundary are divided into smaller triangles.

\subsection{Noise Control in a Car Interior}

As an example application of the method, noise control in a BMW 330i car interior is considered, see Fig. 1(a). The interior of the car excluding the driver is the domain $\Omega(\mathbf{r})$. The objective of the noise control is to minimize the noise in the driver's ears. Thus, $\Xi$ is defined as a set

$$
\Xi(\mathbf{r})=\left\{\mathbf{e}_{l}, \mathbf{e}_{r}\right\} \subset \Omega(\mathbf{r})
$$




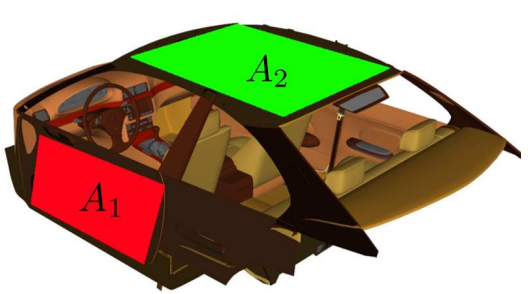

(a)
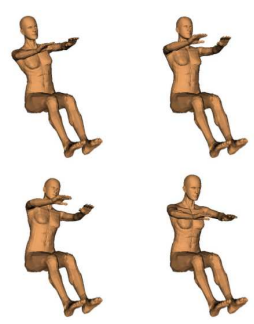

(b)

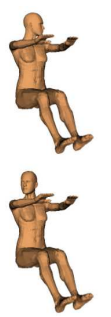

Fig. 1 (a) A three-dimensional model of a BMW 330i car interior. The subdomains $A_{1}$ and $A_{2}$ are marked with red and green colors, respectively. The subdomain $A_{3}$ is located on the right front door, which is not visible. (b) The driver's posture parameters from left to right: $r_{1}$ is the driver's sideways bending, $r_{2}$ is the forward bending, $r_{3}$ is head rotation. In the upper figures, the parameter's lowest value is shown and in the lower figures, the highest value is shown.

where $\mathbf{e}_{l}(\mathbf{r})$ and $\mathbf{e}_{r}(\mathbf{r})$ are the co-ordinates of the left and right ear, respectively. The noise measures (4) and (8) have now expressions

$$
\begin{aligned}
N(\omega, \mathbf{r}, \gamma) & =\left|p\left(\omega, \mathbf{e}_{l}, \mathbf{r}, \gamma\right)\right|^{2}+\left|p\left(\omega, \mathbf{e}_{r}, \mathbf{r}, \gamma\right)\right|^{2} \\
\tilde{N}(\omega, \mathbf{a}, \mathbf{r}, \gamma) & =\left|p\left(\omega, \mathbf{a}, \mathbf{e}_{l}, \mathbf{r}, \gamma\right)\right|+\left|p\left(\omega, \mathbf{a}, \mathbf{e}_{r}, \mathbf{r}, \gamma\right)\right| .
\end{aligned}
$$

It is assumed that there is only the driver and no other passengers or significant objects in the car that would influence the sound propagation. The driver's variable properties like shape and posture are taken into account by considering a stochastic domain in the computation.

The driver is modeled by using the freely available Animorph library, based on [4]. Three parameters to model the driver are considered: $r_{1}$ is the driver's sideways bending angle, $r_{2}$ is the forward bending angle, and $r_{3}$ is the head rotation angle to left/right. These parameters are illustrated in Fig. 1(b) and their discrete values are as follows: $r_{1} \in\{-20,-10,0,10,20\}, r_{2} \in\{-5,0,5,10,15\}$, and $r_{3} \in\{-50,-25,0,25,50\}$. The random variable vector $\mathbf{r}=\left(r_{1}, r_{2}, r_{3}\right)$ determines the posture of the driver.

In the car cabin interior, the noise source is modeled as a uniformly vibrating surface behind the leg room, which is a simplification of the real noise source. There are three possible surfaces where actuators may be located: on the left front door below window $\left(A_{1}\right)$, on the roof $\left(A_{2}\right)$ and on the right front door below window $\left(A_{3}\right)$, see Fig. 1(a). The size of the door subdomains $A_{1,3}$ is $0.35 \times 0.8 \mathrm{~m}^{2}$ and the roof subdomain $1.0 \times 1.0 \mathrm{~m}^{2}$. These subdomains are placed and scaled beside each other so that they form a unit square, which makes it possible to use generic optimization formulation where optimization variables take values between $[0,1]$. If an actuator crosses the boundary of the subdomain that it belongs to, it is cut so that only the part inside the subdomain is considered as an actuator. The anti-noise actuators are let to overlap freely and it also appears that they overlap in many optimized solutions. 
Overlapping could be avoided by penalizing such solutions during the optimization process.

To solve the Helmholtz equation (1) with the finite element method, a collection of meshes consisting of linear tetrahedra and triangles were generated with Ansys ICEM CFD. Each mesh corresponds to a different driver posture and they were generated so that there are at least 10 nodes per wavelength at a $1000 \mathrm{~Hz}$ wave. The total number of meshes is $5^{3}=125$ which is the number of the parameter combinations $\left(r_{1}, r_{2}, r_{3}\right)$.

The study was done in the frequency range $50-500 \mathrm{~Hz}$ with $25 \mathrm{~Hz}$ steps. This means that 18 frequencies were sampled. By employing the reciprocity principle, a sound source was placed in an ear. The acoustic model was solved for all 125 sampled driver's postures for both ears. Thus, discrete Helmholtz equations were solved $125 \cdot 18 \cdot 2=4500$ times for the optimal anti-noise control.

\section{Evolutionary Multi-Objective Optimization}

A general form of a multi-objective minimization problem is

$$
\begin{array}{ll}
\text { minimize } & \left\{f_{1}(\mathbf{x}), f_{2}(\mathbf{x}), \ldots, f_{k}(\mathbf{x})\right\} \\
\text { subject to } & \mathbf{x} \in S,
\end{array}
$$

where $f_{1, \ldots, k}: \mathbb{R}^{n} \rightarrow \mathbb{R}$ are conflicting objective functions that are minimized by altering values of the design variables forming a vector $\mathbf{x} \in \mathbb{R}^{n}$ within a feasible region $S \subset \mathbb{R}^{n}$. The solution $A$ is said to dominate the solution $B$ if all components of $f(A)$ are at least as good as those of components of $f(B)$, with at least one strictly better component. Furthermore, $A$ is non-dominated if it is not dominated by any feasible solution. Correspondingly, the solution $A$ belongs to the Pareto optimal set if it is not dominated by any other feasible solution.

The multi-objective optimization problem for the locations and sizes of anti-noise actuators is defined to maximize the average expected attenuation obtained by local noise control at two frequency ranges simultaneously. The frequency ranges are given by the vectors $\omega=\left(\omega_{1}, \ldots, \omega_{n_{\omega}}\right), \iota=\left(\iota_{1}, \ldots, \iota_{n_{\iota}}\right)$. The objective functions are as follows:

$$
f_{1}(\mathbf{x})=\frac{1}{n_{\omega}} \sum_{i} Q\left(\mathbf{x}, \omega_{i}\right) \quad \text { and } \quad f_{2}(\mathbf{x})=\frac{1}{n_{\iota}} \sum_{i} Q\left(\mathbf{x}, \iota_{i}\right),
$$

where $Q$ is the quality measure (10) and $\mathbf{x}$ is the design vector containing the location co-ordinates and radii of the anti-noise actuators.

Evolutionary multi-objective optimization algorithms (EMOA) (see, e.g., [6]) are among the widely used approaches in solving demanding engineering problems with multiple objectives. Different EMOAs employ various methods in the way they generate trial points and how they bring about the evolution of the population. Usually 
fitness is based primarily on dominance (non-dominated solutions are preferred), and secondarily on diversity (solutions on crowded regions are pruned).

Probably the most often referred and widely utilized algorithm in the abovementioned category is the elitist non-dominated sorting genetic algorithm (NSGAII) [8]. Yet, NSGA-II is claimed to have certain defects both in its performance and in its basic foundations, and for this reason both NSGA-II and one algorithm that should overcome its defects, namely unrestricted population-size EMOA (UPSEMOA) [3], are considered. In the following subsections short descriptions of both of these algorithms are given together with the corresponding parameters that are used in the numerical examples.

\subsection{NSGA-II}

Functioning of the dominance and diversity preservation based NSGA-II algorithm as it is implemented here is described briefly as follows:

Step 1. Create an initial (parent) population of $n_{\text {beginpop }}=10 n_{\text {pop }}=10000$ population members randomly.

Step 2. Evaluate objective function values for the initial population and choose the $n_{\text {pop }}=1000$ best ones based on non-domination.

Step 3. Generate $n_{p o p}$ trial points to create a child population by using the simulated binary cross-over operation (SBX, see [7]). The following parameters have been used: cross-over probability $p_{c}=0.9$, mutation probability $p_{m}=1 / n_{\text {vars }}$ where $n_{\text {vars }}$ is the number of variables, SBX cross-over parameters for the crossover $\eta_{c}=10$, and mutation $\eta_{m}=10$, tournament size $n_{\text {tour }}=2$.

Step 4. Evaluate objective function values for the child population.

Step 5. Combine the parent and the child populations. Identify non-dominated solutions from the combined population. Create the next parent population by taking solutions from the combined population to the new one:

a. If there is excess of non-dominated solutions to fit into the next population, prune such excess solutions which are located in more crowded areas (diversity preservation).

b. If there are not enough non-dominated solutions to fill the next population, identify again non-dominated solutions remaining in the combined population, and continue this cycle until the population is filled.

Step 6. If the number of allowed generations is not exceeded, or the budget for objective function evaluations is not exhausted, go back to Step 3.

Unfortunately, it seems not to be widely fathomed that this type of algorithm suffers from several theoretical drawbacks, such as oscillation [3] (lack of convergence [10]), deterioration of the population, and lack of performance. 
It is said that the method involves oscillation if a solution close to the Pareto optimal set is replaced by another non-dominated solution which improves diversity but is at the same time located much farther from the Pareto optimal set. If in the history of all the evaluated solutions there exist solutions that dominate the solutions in the current population, then the population is said to be deteriorated. If deterioration occurs, it suggests that the algorithm has wasted some objective function evaluations, and could have actually performed better. This behaviour also contributes to general lack of performance.

\subsection{UPS-EMOA}

The basic feature of the recently published UPS-EMOA [3] is the use of a population which has no artificial size limit. Instead, the population always contains all the nondominated solutions found during the optimization process, and thus the population expands. Theoretically, this may lead to a situation where storage requirements are unbounded. In practice, we have not witnessed such behaviour, as the number of evaluations is kept finite. By expanding population, the algorithm overcomes some problems of the current EMO approaches, such as oscillation (lack of convergence), deterioration of the population, and lack of performance. Steps of the UPS-EMOA implementation used in this paper are presented as follows:

Step 1. Initialize the population within the given search space using $n_{\text {beginpop }}=$ 10000 points covering the space as uniformly as possible. Points are created using a space-filling Hammersley sequence [9].

Step 2. Evaluate the objective function values of the new points.

Step 3. Combine the current population with the new points. Identify non-dominated solutions, and move all these to the next population. If the minimum size of the population $n_{\text {min }}=50$ is not reached, take non-dominated solutions from the remaining points, and continue until the minimum size is reached.

Step 4. Select randomly $n_{\text {burst }}=260$ points from the current population to be used as parents. Generate one new child point for every parent point using the point generation mechanism of differential evolution (DE, see [12]), using cross-over probability $C_{r}=0.5$ and the scaling factor $F=1.0$. In the creation of the new point, all points in the current population may participate. Points which are not inside the given search space are truncated to the border, similarly as in NSGA-II.

Step 5. Evaluate the objective function values of the child population, and if the budget for objective function evaluations is not exhausted, go back to Step 3 . 


\subsection{Comparison of EMOAs by Hypervolume Measure}

With multi-objective optimization algorithms that produce an approximation of the Pareto-optimal set, measuring the performance of a given algorithm is far from trivial. To characterize the goodness of the solution set, all solutions should be as close as possible to the real Pareto optimal set (closeness) and the solutions should cover the whole Pareto optimal set as well as possible (diversity), meaning that the distribution of the solutions along the Pareto optimal set should be even, and the extent of the solutions should be as high as possible.

Recently, a hypervolume indicator [17] has gained popularity both as a performance metric and as a selection criterion in EMOAs. The hypervolume defines the volume of the objective space dominated by the given solution set, and as such it can give information about both closeness and diversity at the same time.

In this study, hypervolume is used as a performance metric to make a rough comparison between two selected algorithms.

\section{Numerical Experiments}

Four numerical optimization test cases are considered to demonstrate and analyze the efficiency of the method. All test cases involve local noise control in a car interior (see Fig. 1(a)) as explained in Sect. 3.4. The test cases are as follows:

- Test case \#1: 2 fixed-size actuators (4 design variables)

- Test case \#2: 3 fixed-size actuators (6 design variables)

- Test case \#3: 3 variable-size actuators (9 design variables)

- Test case \#4: 8 variable-size actuators (24 design variables)

Equations (14) are considered as contradicting objective functions, with two frequency ranges 50-275 $\mathrm{Hz}$ and 275-500 $\mathrm{Hz}$ corresponding to vectors $\omega=[50,75, \ldots, 250]$ and $\iota=[275,300, \ldots, 500]$. For the test case \#1, the design vector $\mathbf{x}=\left(x_{1}, x_{2}, r, x_{3}, x_{4}, r\right)$, where $r=0.112 \mathrm{~m}$, i.e. there are four design variables. For the test case \#2, similarly $\mathbf{x}=\left(x_{1}, x_{2}, r, x_{3}, x_{4}, r, x_{5}, x_{6}, r\right)$. For the test cases $\# 3$ and \#4, where the actuator radius $r \in[0.05,0.175] \mathrm{m}$ is also a design variable, $\mathbf{x}=\left(x_{1}, \ldots, x_{n}\right)$, with $n=9$ and $n=24$, respectively. The test cases \#1-\#3 were run until the limit of $100000 \mathrm{ob}-$ jective function evaluations and the test case \#4 was run until the limit of 200000 objective function evaluations.

The first test case was chosen in order to present a simple case with a low number of design variables. The test cases \#2-\#4 present more difficult optimization problems, where EMO approaches may not be able to find a global unambiguous optimum, which is a well-known feature of the used methods when the search space is large due to the number of design variables and when there are plenty of local minima in the problem. Nevertheless, these methods are able to bring about a significant improvement, when compared with a sophisticated engineer guess. 

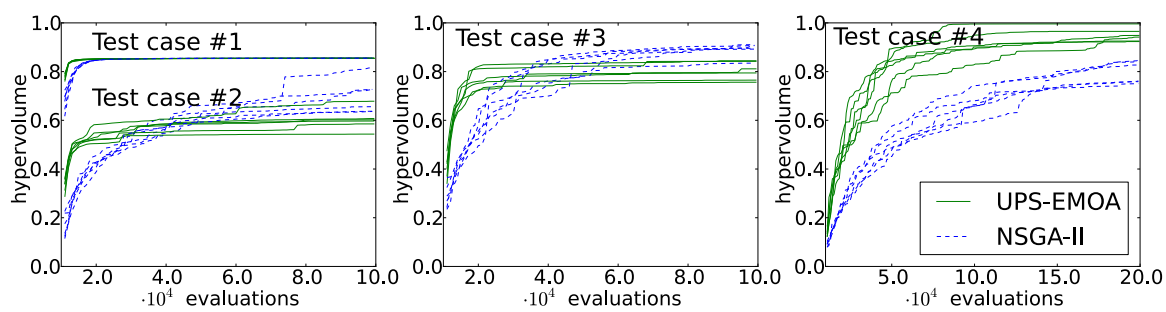

Fig. 2 Hypervolume as a function of the number of objective function evaluations with UPSEMOA and NSGA-II. Six random number generator seed numbers for each test case and algorithm. The resulting lines of different test cases should not be compared to each other, due to incompatible scales.
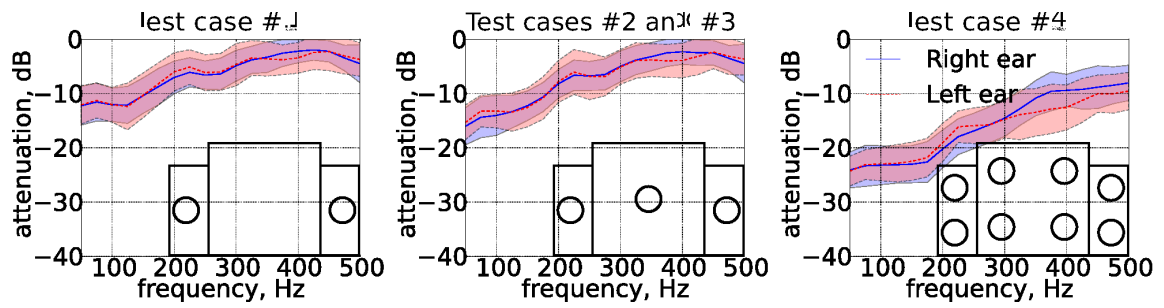

Fig. 3 Engineer guesses of good anti-noise actuator configurations. The figures show (1) the antinoise actuator configuration in the subdomains $A_{1}, A_{2}$, and $A_{3}$ (see Fig. 1(a)), and (2) the expected value of attenuation in the left and right ear with standard deviation (the shaded region). Corresponding objective function values of (10) are plotted in Fig. 4.

\subsection{Convergence of Multi-Objective Optimization Methods}

To justify the choice of using UPS-EMOA as a preferred optimization algorithm for the presented problem, the convergence was compared to the NSGA-II by evaluating hypervolumes of the solution fronts (see Sect. 4.3). The hypervolume as a function of the number of objective function evaluations is plotted for all test cases in Fig. 2.

For the test case \#1, UPS-EMOA converges notably faster to its maximum, already at 14000 evaluations, while NSGA-II reaches the same level at 20000 evaluations. This is the only test case where robust convergence towards the identical solution front is obtained and it is due to the low number of design variables, $n_{\text {vars }}=4$. For all test cases, it is clearly seen that UPS-EMOA convergence is notably faster in the beginning of the optimization process. However, none of the test cases \#2-\#4 converge robustly towards a single solution front, which is due to the larger search space with plenty of local minima.

For the test cases \#2 and \#3, NSGA-II eventually finds better solution fronts, despite its slower convergence in the beginning. In Fig. 5, the final solution fronts after 99440 objective function evaluations are plotted, where it can clearly be seen that while the right part of the front is identical, on the left part the NSGA-II has progressed further. We suggest that this is due to concentrated point density of the UPS-EMOA results on the right part of the front, leading to a situation where points 


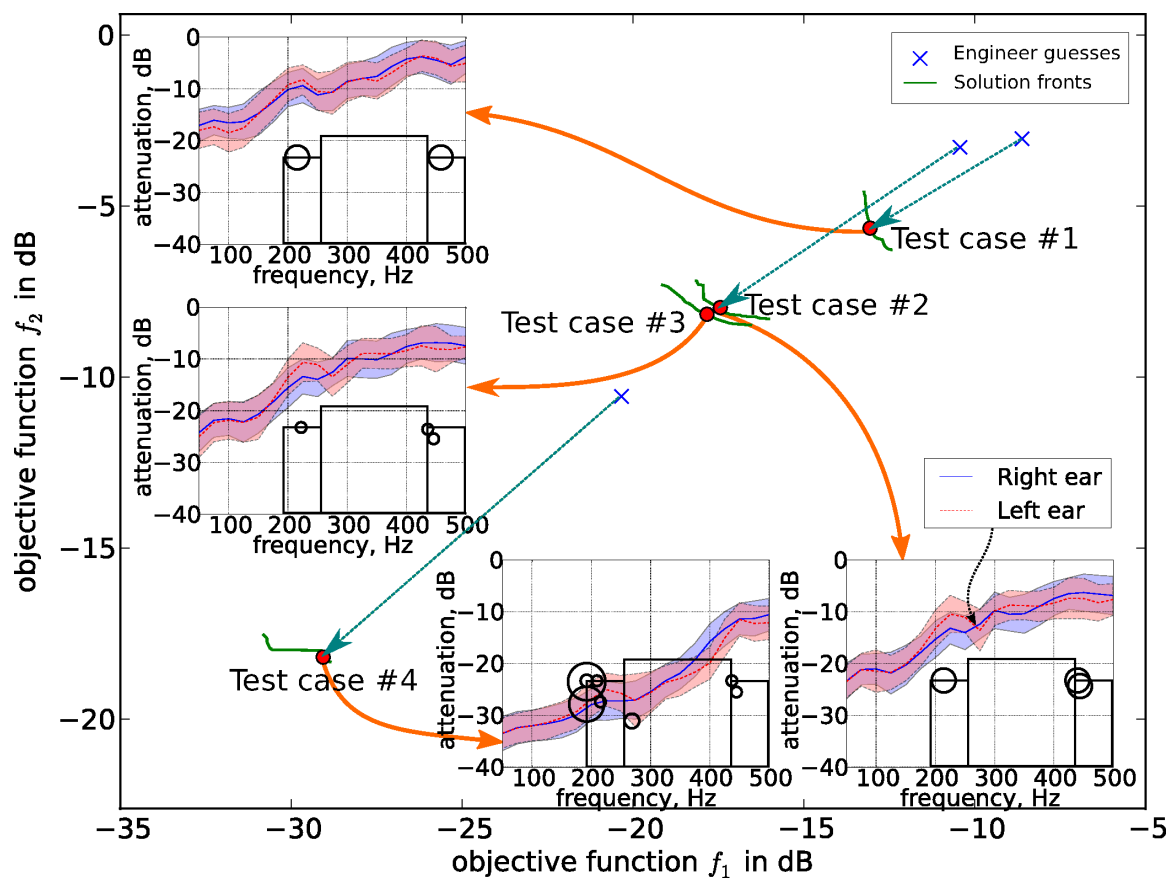

Fig. 4 The solution fronts for all test cases obtained by UPS-EMOA. Blue crosses correspond to non-optimized engineer guesses, shown in Fig. 3. One solution from each front is selected and shown in small subfigures, similarly as in Fig. 3.

on the left have only diminishing probability to be selected as parents. Thus the development of the front in that region suffers.

For the test case \#4, where there are 24 design variables and for all runs, UPSEMOA converges faster and gives better solution fronts than NSGA-II. As a conclusion, UPS-EMOA is clearly a better choice, when the CPU time usage is limited and/or when a larger number of design variables is involved.

\subsection{Example Solutions}

In Fig. 4, the solution fronts for all test cases are shown, obtained by UPS-EMOA. These fronts can be compared to the objective function values obtained by sophisticated engineer guesses (see Fig. 3) that are plotted as well. It is clearly seen that optimization improves the noise control remarkably. The figure also illustrates the big improvement obtained when the number of anti-noise actuators is increased; compare the solution fronts for the test cases \#1 to \#2 where the number of actuators increases from 2 to 3 ( $\sim 3-5 \mathrm{~dB}$ improvement in both objective function values), 


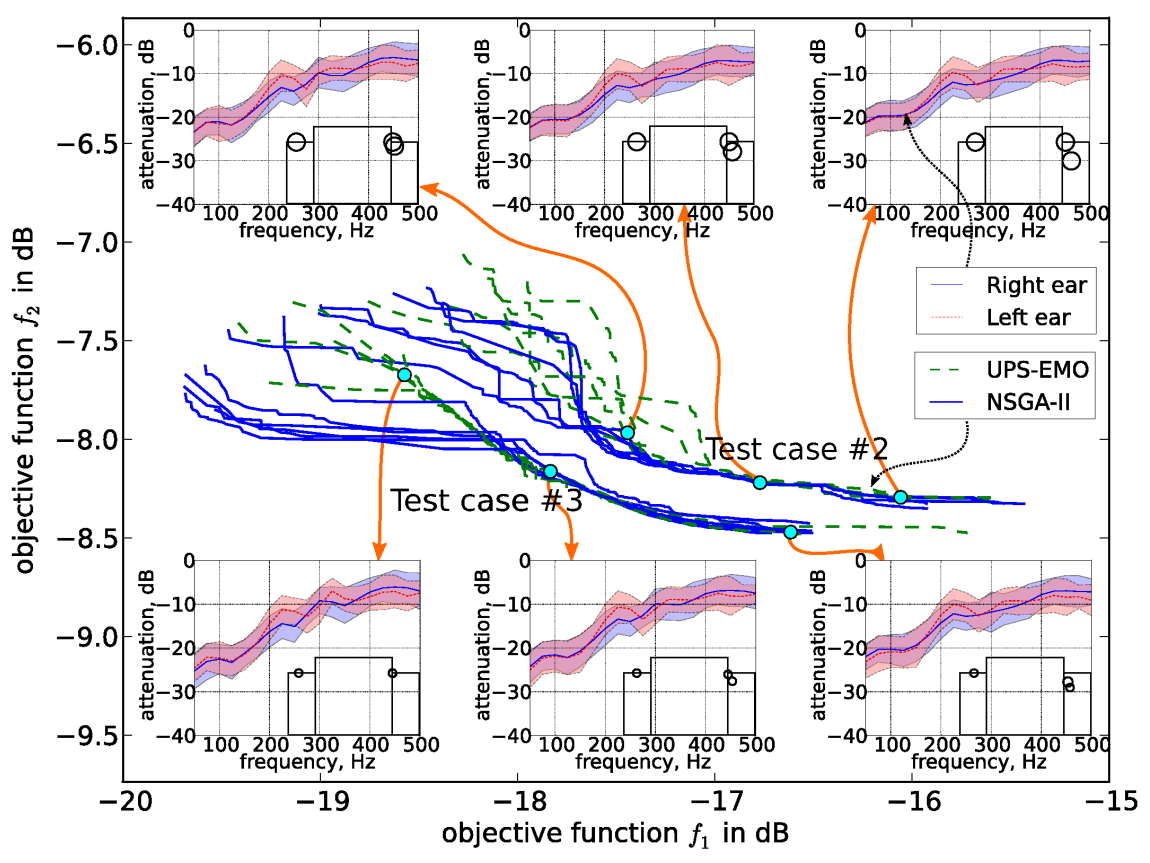

Fig. 5 The solution fronts for the test problems \#2 and \#3 obtained by NSGA-II and UPS-EMOA, six random number generator seed numbers. Three solutions (cyan circles) from a single UPSEMO front are selected for both test cases and they are shown in small subfigures, similarly as in Fig. 3.

and the solution fronts for the test cases \#3 to \#4, where the number of actuators increases from 3 to 8 ( $\sim 10 \mathrm{~dB}$ improvement in both objective function values).

In Fig. 5, the solution fronts for the test cases \#2 and \#3 are given after 99440 objective function evaluations. Three solutions are selected for both test cases from a single front obtained by UPS-EMOA. Both test cases have three anti-noise actuators, but the difference between them is that while in the test case $\# 2$, the sizes (radii) of each actuator are constant, in the test case \#3 they may vary. It is seen that this increase in the degree of freedom gives only a $0.2-0.5 \mathrm{~dB}$ enhancement in objective function values. It is also seen that smaller anti-noise actuators seem to be preferable in this case. Similar behavior in actuator placements is seen in both test cases.

\section{Conclusions}

A novel technique was proposed to find optimal locations for anti-noise actuators by using a finite element model based numerical evaluation method for optimal local 
noise control. The optimization of anti-noise actuator configuration, i.e. the placing and size of each actuator, was formulated as a multi-objective optimization problem so that optimal noise reduction at two frequency ranges could be obtained.

As an example problem, local noise control in a car interior with a driver in varying postures was considered and numerical results were presented. Two evolutionary multi-objective algorithms, UPS-EMOA and NSGA-II were used as optimization methods and their performance was compared. It was found that in all test cases UPS-EMOA was converging faster in the beginning of the optimization process, but NSGA-II was able to give better final solution fronts in two test cases.

Numerical examples clearly demonstrated that, by employing optimization of anti-noise actuator configuration, it is possible to obtain a significant improvement in the objective function values over sophisticated engineer guesses.

Acknowledgements The research was funded by Academy of Finland, the grant \#250979.

\section{References}

1. T. Airaksinen, E. Heikkola, and J. Toivanen. Local control of sound in stochastic domains based on finite element models. J. Comput. Acoust., 19(2):205-219, 2011.

2. T. Airaksinen, A. Pennanen, and J. Toivanen. A damping preconditioner for time-harmonic wave equations in fluid and elastic material. J. Comput. Phys., 228(5):1466-1479, 2009.

3. T Aittokoski and K Miettinen. Efficient evolutionary approach to approximate the Paretooptimal set in multiobjective optimization, UPS-EMOA. Optimization Methods and Software, 25(6):841-858, 2010.

4. M. Bastioni, S. Re, and S. Misra. Ideas and methods for modeling $3 \mathrm{~d}$ human figures. In R. K. Shyamasundar, editor, Proceedings of the 1st Bangalore Annual Compute Conference. ACM, 2008. doi:10.1145/1341771.1341782.

5. A. Bermudez, P. Gamallo, and R. Rodriguez. Finite element methods in local active control of sound. SIAM J. Control Optim., 43(2):437-465, 2004.

6. K. Deb. Multi-Objective Optimization using Evolutionary Algorithms. John Wiley \& Sons, Ltd., Chichester, England, 2001.

7. K. Deb and R. B. Agrawal. Simulated binary crossover for continuous search space. Complex Systems, 9(2):115-148, April 1995.

8. K Deb, A Pratap, S Agarwal, and T Meyarivan. A fast and elitist multiobjective genetic algorithm: NSGA-II. IEEE Transactions on Evolutionary Computation, 6(2):182-197, APR 2002.

9. J. Hammersley. Monte carlo methods for solving multivariable problems. In Proceedings of the New York Academy of Science, volume 86, pages 844-874, 1960.

10. M. Laumans, L Thiele, K. Deb, and E. Zitzler. Combining convergence and diversity in evolutionary multi-objective optimization. Evolutionary Computation, 10(3):263-282, 2002.

11. P. A. Nelson and S. J. Elliot. Active Control of Sound. Academic Press, London, 1999.

12. K. V. Price, R. M. Storn, and J. A. Lampinen. Differential Evolution - A Practical Approach to Global Optimization. Springer-Verlag, Berlin, 2005.

13. C. G. Provatidis, S. T. Mouzakitis, and G. N. Charalampopoulos. Simulation of active noise control in enclosures using direct sound field prediction. J. Comput. Acoust., 17(1):83-107, 2009.

14. P. Shorter. Recent advances in automotive interior noise prediction. SAE Technical Paper 2008-36-0592, 2008. doi:10.4271/2008-36-0592. 
15. D. A. Stanef, C. H. Hansen, and R. C. Morgans. Active control analysis of mining vehicle cabin noise using finite element modelling. J. Sound Vib., 277(1-2):277-297, 2004.

16. L. L. Thompson. A review of finite-element methods for time-harmonic acoustics. J. Acoust. Soc. Am., 119(3):1315-1330, 2006.

17. E Zitzler and L. Thiele. Multiobjective optimization using evolutionary algorithms - a comparative case study. In Conference on Parallel Problem Solving from Nature (PPSN V), pages 292-301, Amsterdam, 1998. 
